\title{
Cervical Necrotizing Fasciitis Caused by Dental Infection
}

\author{
Chi-Woong Song, Hyun-Joong Yoon, Da-Woon Jung, Sang-Hwa Lee \\ Department of Oral and Maxillofacial Surgery, The Catholic University of Korea, Yeouido St. Mary's Hospital
}

\begin{abstract}
Necrotizing fasciitis (NF) is defined as rapidly progressive necrosis of subcutaneous fat and fascia. Although NF of the face is rare, its mortality rate is nearly $30 \%$. It usually originates from dental infection and can lead to involvement of the neck, mediastinum, and chest wall. Complications resulting from pre-existing systemic diseases can increase the mortality rate. Known complication factors for $\mathrm{NF}$ include diabetes, malnutrition, advanced age, peripheral vascular disease, renal failure, and obesity. Here, we report a case of NF originating from dental infection in an 88-year-old woman already diagnosed with hypertension, thoracic aortic aneurysm, and renal diseases. Such conditions limited adequate surgical and antibiotic treatment. However, interdisciplinary treatment involving multiple departments was implemented with good results.
\end{abstract}

Key words: Necrotizing fasciitis, Thoracic aortic aneurysm

\section{Introduction}

Necrotizing fasciitis (NF) is defined as rapidly progressive necrosis of subcutaneous fat and fascia. It is a life-threatening infection characterized by progressive, rapid necrosis of subcutaneous tissues and fascial planes. NF of the face is rare and usually originates from dental infection, and the mortality rate is nearly 30\%[1]. The probability of death among patients with NF due to infectious causes is $14 \%$, and common causes of death are usually complications related to underlying systemic diseases. Such complications arise due to cardiopulmonary diseases, diabetes mellitus (DM), cancer, hepatic failure, renal disease, hypertension, Methicillin-Resistant Staphylococcus aureus
(MRSA), and age[2,3]. It is very important to check patients' medical history when treating oral and maxillofacial infections. Elderly patients are more at risk in terms of underlying systemic diseases and are more likely to develop complications. The major complication factor when treating infections is DM. In the case of DM, patients' metabolic regulation can be altered by surgery, and delayed wound healing can increase the risk of infection. Further, the immune systems of older DM patients usually become weak with advanced age. A weak immune system can increase the risk of spreading infections, thus leading to complications[2,3]. Another complication factor is renal failure, as antibiotics are excreted by the kidneys. Nephrotoxins are present in aminoglycosides and anti-

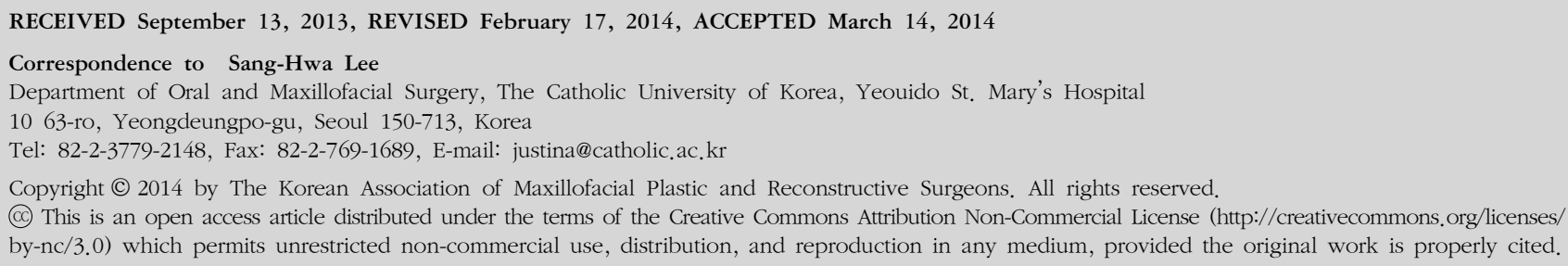


biotics such as vancomycin. Therefore, the use of antibiotics in patients with renal failure is limited since it can cause complications such as acute kidney injury (AKI) or chronic kidney disease (CKD)[4]. Occurrence of NF in patients with thoracic aortic aneurysms is normally very rare but is quite common in elderly patients. Under stress, simple treatments can lead to a ruptured aortic aneurysm, resulting in patient death[5]. Aneurysm of the thoracic aorta causes local enlargement of the thoracic aorta, resulting in ruptures as the disease progresses. Enlargement of the aorta has been shown to be related to higher risk of rupture[6]. It is also known that intramural hematomas are caused by ruptures in the Vasa vasorum. These occur naturally in elderly patients or in patients with hypertension for the most part[7]. We successfully treated an 88-year-old female patient presenting with several underlying diseases, including aortic aneurysms, vascular wall hemorrhages, and hypertension, who had developed NF as well as myonecrosis on the left side of the face due to a dental infection. We successfully administered multidisciplinary treatments combining the departments of thoracic surgery, infection, and nephrology. We report this case along with a review of the literature.

\section{Case Report}

An 88-year-old female patient presenting with swelling on the left side of the face for several months was referred to the Department of Oral and Maxillofacial Surgery, Yeouido St. Mary's Hospital. This patient had visited a local dental office but was not able to receive treatment due to the occurrence of systemic diseases. Swelling on the left side of the face had been present since March 30th, 2012. The patient had applied honey to the affected

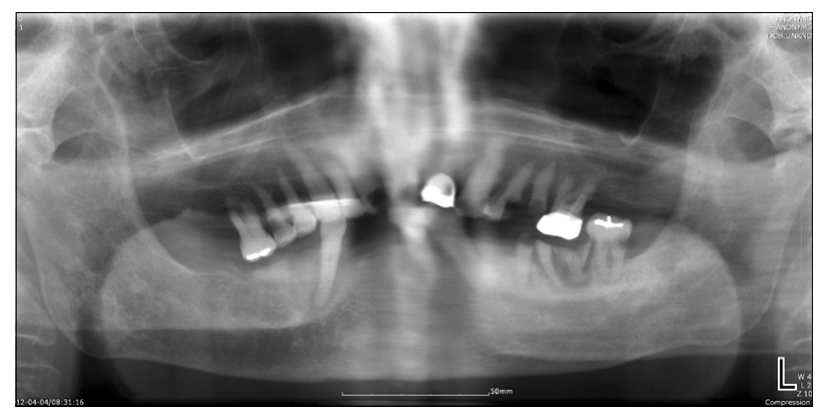

Fig. 1. Dental panoramic view. area as a folk remedy, but this only made symptoms more severe. Thus, the patient sought medical attention at the emergency room on April 4th, 2012. The medical history of this elderly patient included hypertension, aneurysm of the thoracic aorta, intramural hematoma, and renal failure.

\section{Diagnosis}

The patient presented with edema and aches on the left side of the face and cervical region, along with complaints of dyspnea, dysphagia, and trismus. Oral examination showed multiple caries, periodontal disease, and gingival edema of the left mandible (Fig. 1). Complete blood cell counts, vital check-up, and computed tomography (CT) were carried out. Vital check showed a blood pressure (BP) of $108 / 54 \mathrm{mmHg}$, which was controlled since the patient had been taking antihypertension medication. Pulse was 78 times/min. Laboratory investigation revealed increased white blood cell counts, C-reactive protein, seg-neutrophils, urea nitrogen, and creatinine levels as well as decreased hemoglobin and lymphocyte levels (Table 1).

Neck CT (enhance) showed abscesses in the left buccal space along with air bubbles in left buccal region's fascial plane. Masseter and pterygoid muscles in the adjacent left masticator space also showed swelling and necrotizing myositis. Left buccal and masticator spaces contained diffuse fat infiltration and cellulitis, whereas the left submandibular gland showed swelling due to increased inflammation. Posterior neck area showed cellulitis, but the airway seemed clean (Fig. 2). The patient was diagnosed with abscesses, NF, and myositis in the left buccal and pterygomandibular spaces based on physical examination, laboratory findings, and radiological imaging.

\section{Treatment}

Triple-antibiotic regimens are usually administered for

Table 1. Results of laboratory investigation

\begin{tabular}{lrc}
\hline \multicolumn{1}{c}{ Investigation } & Result & Normal range \\
\hline Hemoglobin $(\mathrm{g} / \mathrm{dL})$ & 10.00 & $12.0 \sim 16.0$ \\
White cell count $\left(\times 10^{9} / \mathrm{L}\right)$ & 19.91 & $5.0 \sim 10.0$ \\
Seg-neutrophils $(\%)$ & 93.90 & $55.0 \sim 75.0$ \\
Lymphocytes $(\%)$ & 3.70 & $20.0 \sim 44.0$ \\
Platelet count $\left(\times 10^{9} / \mathrm{L}\right)$ & 143.00 & $150.0 \sim 450.0$ \\
Blood urea nitrogen (mg/dL) & 44.80 & $8.0 \sim 23.0$ \\
Creatinine (mg/dL) & 2.07 & $0.5 \sim 1.2$ \\
C-reactive protein $(\mathrm{mg} / \mathrm{L})$ & 360.36 & $0.1 \sim 5.0$ \\
\hline
\end{tabular}



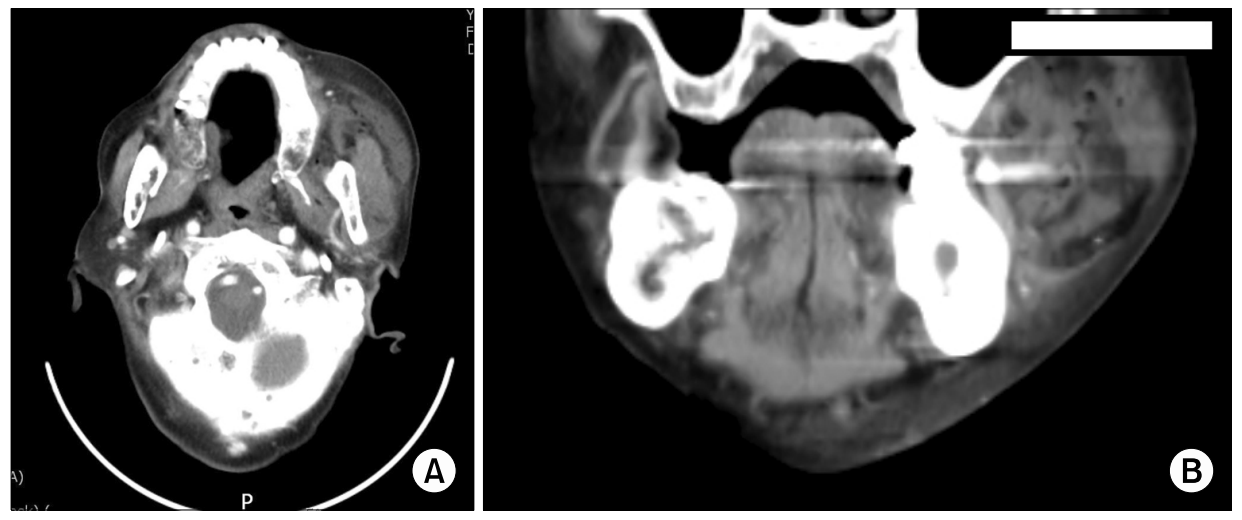

Fig. 2. Necrotizing fasciitis on left buccal region. (A) Axial view; (B) Coronal view.

the treatment of deep infections on the maxillofacial area. In the present case, amoxicillin/clavulanic acid, gentamycin, and metronidazole were administered before antimicrobial susceptibility tests. Many factors must be considered when applying antibiotics after surgical drainage. In general, antimicrobial susceptibility tests after separation of cariogenic bacilli are used to determine which types of antibiotics to use. In the present case, cultured pus from the patient contained Gram positive cocci, Gram positive bacilli, mid-grade Gram positive rod-shaped bacteria, and minor-grade $S$, aureus. These cariogenic bacilli were used in the antimicrobial susceptibility tests (Table 2). The patient was subjected to restrictions in usage of antibiotics due to renal failure. CT results from 2008 showed both shrinkage of both kidneys, and urinalysis revealed 2.07 $\mathrm{mg} / \mathrm{dL}$ of creatinine, Cr-eGFR 24.06. This infection combined with the patient's use of nonsteroidal anti-inflammatory drugs and contrast medium increased the risk of developing AKI and CKD. Limited amounts of augmentin, metronidazole, and aminoglycoside were administered after careful consultation with the departments of infectious diseases and nephrology. The patient was given a combination of amoxicillin with clavulanic acid (0.6 g twice a day), gentamycin (160 mg every 48 hours), and metronidazole (500 mg three times a day). Augmentin and gentamycin were administered in a lose-dose regimen, whereas metronidazole was treated normally. After 7 days, metronidazole treatment was stopped. Pus culture confirmed the presence of MRSA. After consultation with the department of infectious diseases, the patient's antibiotic regimen was changed to vancomycin, and treatment with augmentin and gentamycin was stopped. The patient was given $1 \mathrm{~g}$ of
Table 2. Bacterial culture and identification (pus)

\begin{tabular}{cl}
\hline Staphylococcus aureus & Identification \\
\hline Ciprofloxacin & Sensitive \\
Clindamycin & Resistance \\
Erythromycin & Resistance \\
Gentamycin & Sensitive \\
Penicillin & Resistance \\
Vancomycin & Sensitive \\
\hline
\end{tabular}

vancomycin intravenously everyday. Vancomycin has nephrotoxic effects, so its dosage was reduced by half.

Surgical drainage through an extraoral incision under general anesthesia was planned. However, the patient was at high risk for dramatic BP changes due a history of systemic diseases, potentially leading to aortic rupture and expiration during surgery under general anesthesia. In fact, the patient was designated as Class III in the American Society of Anesthesiologists classification system upon whole body assessment. In addition, the patient already showed symptoms of NF, thereby increasing the potential of dermolysis upon extraoral incision. As a result, surgical drainage through an intraoral incision under local anesthesia was carried out under the supervision of an anesthesiologist. Remifentanil was administered to relieve pain. Nicardipine was used to manage BP. During the operation, the patient's BP was 167/100. After surgery, the patient was admitted to the intensive care unit and was carefully followed-up by doctors from the departments of thoracic surgery, nephrology, infection, and ear, nose, throat. The patient experienced increased pain and was given pethidine. Vital signs were checked each hours, and the patient was put on an oxygenator.

Intraoral dressing was performed twice a day. $\mathrm{SpO}_{2}$ lev- 
els and BP were carefully monitored. During the procedure, pethidine was administered to control pain and reduce stress. So we pretended minimize the risk of heart damage. Since it was impossible to perform a CT scan through the contrast medium, CT was carried out without any medium. CT taken 2 days after surgery showed reduced abscess number in the left buccal region, and air spaces in the leftbuccal and masticator spaces were also reduced. Therefore, additional intraoral drainage was performed in the manner as the prior incision and drainage.

Continuous administration of antibiotics and intraoral dressing contributed to improvement of the patient's symptoms, and edema on the left side of the face gradually disappeared. Exactly 21 days after surgery, extractions of \#24, 25, 26, 32, 34, 35, 36, and 37 were performed under intravenous sedation with propofol in order to remove the origin of infection. Extractions of $\# 11,21$, and 22 were performed on May 7th, 2012 (33 days after surgery) while extractions of $\# 15,16$, and 43 were performed on May 16th. On May 19th, 2012, most abscess pockets had disappeared and removal of the origin of infection had finished. As clinical conditions were favorable, the patient was discharged from the hospital.

\section{Discussion}

NF was first described in 1848. Wilson introduced the term NF in 1952 and found no specific pathologic bacteria related to the disease[8]. NF is a destructive and potentially fatal soft tissue infection characterized by extensive necrosis and gas formation in subcutaneous tissues and fascia, with serious involvement of the muscles, vessels, nerves, and fat. However, in the maxillofacial region, NF is less common. The process is often the result of a dental infection supported by aerobic and anaerobic bacteria[9]. A patient will suffer fever, chills, tachycardia, malaise, altered levels of consciousness, and toxic appearance. There are two types of NF: type I, mixed infection of anaerobes plus facultative species such as Streptococci or Enterobacteriaceae; and type II, infection with group A Streptococci[1]. In this case, the patient was classified as having type I NF based on pus culture. The mortality rate of patients with NF varies significantly from $10 \%$ to $40 \%$, and figures as high as $80 \%$ have been reported when early surgical or medical inter- vention is not available[10]. Due to the rarity of the disease, diagnosis and treatment are often delayed, which may result in a fatal outcome due to respiratory problems or systemic complications[9]. Early recognition of this life-threatening disease is crucial in the initiation of successful treatment. Aggressive surgical intervention, intravenous wide-spectrum antibiotics, and supportive therapy are the most accepted treatment modalities for the successful management of $\mathrm{NF}[8-10]$.

Complication factors such as advanced age and DM facilitate the progression of infections to deeper structures. The mortality rate of NF is dependent upon these complication factors[11]. DM is the most common underlying disease behind NF progression, and it alone accounted for $46 \%$ to $72 \%$ of NF cases in a previous study[12]. Specifically, DM presents in $32 \%$ to $66 \%$ of all NF cases as well as adversely affects the intracellular oxidative destruction of pathogens, neutrophil adhesion, chemotaxis, and cellular immunity[13]. Advanced age has long been implicated in increased mortality in patients with NF, and most studies agree that patients older than 50 years of age are at increased risk of death[14]. Renal failure, which is known to predispose individuals to invasive streptococcal infections, is also associated with alteration of the immune system[15]. Before any operation, all NF patients undergo aggressive fluid resuscitation and are treated with parenteral broad-spectrum antimicrobial agents according to renal status[11]. One of the most fearful side effects of antibiotics is nephrotoxicity, such as AKI or CKD. It is estimated that acute renal failure is associated with mortality rates exceeding 50\% in critically ill patients[4]. Streptococcal toxic shock syndrome is another severe complication factor that is strongly associated with mortality[16]. The overall mortality rate of $S$. aureus-induced NF is 33\%, whereas the mortality rate of MRSA-induced NF can reach up to $50 \%$. Vancomycin or teicoplanin is often administered in MRSA-induced NF cases[3]. Additionally, complication factors include cardiovascular diseases, obesity, liver cirrhosis, and hypertension[17].

However, mortality rates of older patients with systemic diseases can be reduced by appropriate treatment modalities resulting from progress in modern technology and medicine. Especially, oral and maxillofacial surgeons in general hospitals receive many geriatric patients from other 
departments after their cavities and periodontitis worsen due to dental infection. Therefore, when treating dental patients with systemic diseases, it is important to consider any latent problems or possible complications as well as work closely with other physicians in related departments.

A thoracic aortic aneurysm causes local enlargement of the thoracic aorta, and it is classified as ascending, aortic arch, or descending based on the occurring region. Further, it is reported to be caused by hypertension. An ascending aortic aneurysm can cause superior vena cava syndrome due to pressure on the superior vena cava or compression necrosis on the breastbone and adjacent rib due to pressure on the posterior region of the breastbone. Aortic aneurysm worsens as time progresses and eventually leads to rupture. This is the most common cause of death in patients with a thoracic aortic aneurysm. The most important predictive factor of rupture is the size of aorta at the time of diagnosis. If the aortic diameter is larger than $6 \mathrm{~cm}$, surgery is recommended since there is increased risk of rupture[5,6]. Other factors include hypertension, Marfan syndrome, trauma, and atherosclerosis[18]. Most aortic aneurysm patients also have hypertension, and it is important to maintain patient BP below 120/80 mmHg in order to prevent disease progress and aortic rupture[19]. Intramural hematomas are caused by rupture of the Vasa vasorum. This occurs naturally in elderly patients or in patients with hypertension, and immediate surgical intervention is usually unnecessary[7]. Such patients with systemic diseases are at a higher risk of aortic rupture when tension is applied to certain areas under general anesthesia. In addition, those with hypertension can experience increases in BP due to tension as well as pain following surgical procedures, leading to possible rupture[20]. Although NF in patients with thoracic aortic aneurysms is rare, pain or aortic aneurysms leading to death can occur.

Despite widespread edema, facial space abscesses, and $\mathrm{NF}$ on the left facial area, we were unable to administer aggressive treatment due to the patient's old age and presence of systemic diseases such as thoracic aortic aneurysm. We were only able to perform surgical drainage through an intraoral incision under local anesthesia. Pethidine was administered 30 minutes before dressing. Analgesics can lower the risk of aortic rupture. During dressing, $\mathrm{SpO}_{2}$ and BP levels were carefully monitored.
Another limitation was the inability to use antibiotics due to the patient's renal failure and MRSA infection. We consulted with physicians in the departments of infectious diseases and nephrology. Antibiotic therapy using adjusted doses of vancomycin was performed, which prevented severe complications. The patient also did not have DM, a potential complication factor that prevents healing. Thus, the patient was properly diagnosed, treated with an interdisciplinary modality, cured, and discharged.

\section{References}

1. Yadav S, Verma A, Sachdeva A. Facial necrotizing fasciitis from an odontogenic infection. Oral Surg Oral Med Oral Pathol Oral Radiol 2012;113:e1-4.

2. Panda NK, Simhadri S, Sridhara SR. Cervicofacial necrotizing fasciitis: can we expect a favourable outcome? J Laryngol Otol 2004;118:771-7.

3. Cheng NC, Wang JT, Chang SC, Tai HC, Tang YB. Necrotizing fasciitis caused by Staphylococcus aureus: the emergence of methicillin-resistant strains. Ann Plast Surg 2011;67:632-6.

4. França MS, Lima SS, Duclou CN, Goulart EM, Romanelli RM. Antimicrobials and renal failure in neutropenic patients. Braz J Infect Dis 2013;17:487-90.

5. Fossaceca R, Guzzardi G, Cerini P, et al. Endovascular treatment of thoracic aortic aneurysm: a single-center experience. Ann Vasc Surg 2013;27:1020-8.

6. Azadani AN, Chitsaz S, Mannion A, et al. Biomechanical properties of human ascending thoracic aortic aneurysms. Ann Thorac Surg 2013;96:50-8.

7. Kruse MJ, Johnson PT, Fishman EK, Zimmerman SL. Aortic intramural hematoma: review of high-risk imaging features. J Cardiovasc Comput Tomogr 2013;7:267-72.

8. Tung-Yiu W, Jehn-Shyun H, Ching-Hung C, Hung-An C. Cervical necrotizing fasciitis of odontogenic origin: a report of 11 cases. J Oral Maxillofac Surg 2000;58:1347-52.

9. Cutilli T, Cargini P, Placidi D, Corbacelli A. Necrotizing fasciitis of the maxillofacial region caused by dental infection. A case report and review. Minerva Stomatol 2007;56:469-76.

10. Dhaif G, Al-Saati A, Ashoor BM. Management dilemma of cervicofacial necrotizing fasciitis. J Bahrain Med Soc 2009;21: 223-7.

11. Oncul O, Erenoglu C, Top C, et al. Necrotizing fasciitis: a life-threatening clinical disorder in uncontrolled type 2 diabetic patients. Diabetes Res Clin Pract 2008;80:218-23.

12. Wong $\mathrm{CH}$, Chang HC, Pasupathy S, Khin LW, Tan JL, Low CO. Necrotizing fasciitis: clinical presentation, microbiology, and determinants of mortality. J Bone Joint Surg Am 2003; 85:1454-60

13. Rajbhandari SM, Wilson RM. Unusual infections in diabetes. Diabetes Res Clin Pract 1998;39:123-8.

14. Golger A, Ching S, Goldsmith $\mathrm{CH}$, Pennie RA, Bain JR. Mortality in patients with necrotizing fasciitis. Plast Reconstr Surg 2007;119:1803-7. 
15. Chong AH, Burrows NP. Fatal group A streptococcal necrotizing fasciitis and toxic shock syndrome in a patient with psoriasis and chronic renal impairment. Australas J Dermatol 2002;43:194-8.

16. Haywood CT, McGeer A, Low DE. Clinical experience with 20 cases of group A streptococcus necrotizing fasciitis and myonecrosis: 1995 to 1997. Plast Reconstr Surg 1999;103: 1567-73.

17. Suwantarat N, Chow DC, Koss W, Lin D, Tice AD. Histologically confirmed necrotizing fasciitis: risk factors, microbiology, and mortality in Hawaii. Int J Infect Dis 2012;16: e886-7.

18. Wilson SK, Hutchins GM. Aortic dissecting aneurysms: causative factors in 204 subjects. Arch Pathol Lab Med 1982;106: 175-80.

19. Choi CH, Kim YA, Seo YS, Choi SJ, Yang SH, Kim JH. A case of emergent cesarean delivery followed by graft replacement of hemiaortic arch in a patient with ruptured thoracic aortic aneurysm. Korean J Obstet Gynecol 2003;46: 1813-17.

20. The Korean Society of Anesthesiologists. Anesthesia and pain management. 2nd ed. Seoul: Elsevier Korea; 2009. p.1480. 